\title{
Early afterglow, magnetized central engine, and a quasi-universal jet configuration for long GRBs
}

\author{
Bing Zhang ${ }^{1}$, Shiho Kobayashi ${ }^{1,2}$, Peter Mészáros ${ }^{1,2,3}$, \\ Nicole M. Lloyd-Ronning ${ }^{4}$ and Xinyu Dai ${ }^{1}$ \\ ${ }^{1}$ Department of Astronomy \& Astrophysics, Penn State University, University Park, PA 16802, \\ USA, \\ ${ }^{2}$ Department of Physics, Penn State University, University Park, PA 16802, USA \\ ${ }^{3}$ The Institute for Advanced Study, Princeton, NJ 08540, USA \\ ${ }^{4}$ Los Alamos National Laboratory, MS B244, Los Alamos, NM 87544, USA
}

\begin{abstract}
Two separate topics are discussed. (1) We describe the classifications of the long GRB early afterglow lightcurves within the framework of the fireball shock model, focusing on the interplay between the reverse and forward shock emission components. We will also provide evidence that the central engine of at least two bursts are entrained with strong magnetic fields, and discuss the implications of this result for our understanding of the GRB phenomenon; (2) We argue that the current gamma-ray burst (GRB) and X-ray flash (XRF) data are consistent with a picture that all GRB-XRF jets are structured and quasi-universal, with a typical Gaussian-like jet structure.
\end{abstract}

\section{EARLY AFTERGLOWS}

\section{Classifications}

A GRB fireball is eventually decelerated by an ambient medium. During the deceleration, a long-lived forward shock propagates into the medium, and a short-lived reverse shock propagates into the fireball shell[1]. The former is responsible for the long-term afterglow emission, while the latter contributes a noticeable emission component at the very early afterglow epoch[1, 2, 3]. So a GRB early afterglow is the interplay between the reverse and the forward shock emission components, and its diagnose would reveal rich information about the GRB fireball and the ambient medium. Very early optical afterglows have now been detected for a handful of GRBs [4, 5, 6], and the Swift GRB mission, scheduled to be launched in June 2004, will greatly increase the sample of the GRB early afterglow data. Here we discuss the classifications of the early afterglow lightcurves within the framework of the fireball shock model. The predictions will be fully confronted by the future abundant early afterglow data.

In general, the early afterglow lightcurves can be categorized according to the type of the ambient medium. Two well-discussed types of medium include a constant density medium ( $n=\mathrm{const}$ ) which is applicable for interstellar medium (ISM), and a windtype medium $\left(n \propto r^{-2}\right)$, which is typical for the environment of a pre-burst massive star progenitor. The left panel of Figure 1 outlines the typical optical early afterglow 
lightcurves for ISM (bottom) and wind (top) cases, respectively[7]. In both cases, the reverse shock emission component peaks at the time when the reverse shock crosses the shell, and drops rapidly after the peak. For the forward shock component, there is a peak for the ISM case corresponding to the crossing of the typical synchrotron frequency across the band [8, 9], while for the wind case, the flux fades exclusively[10].
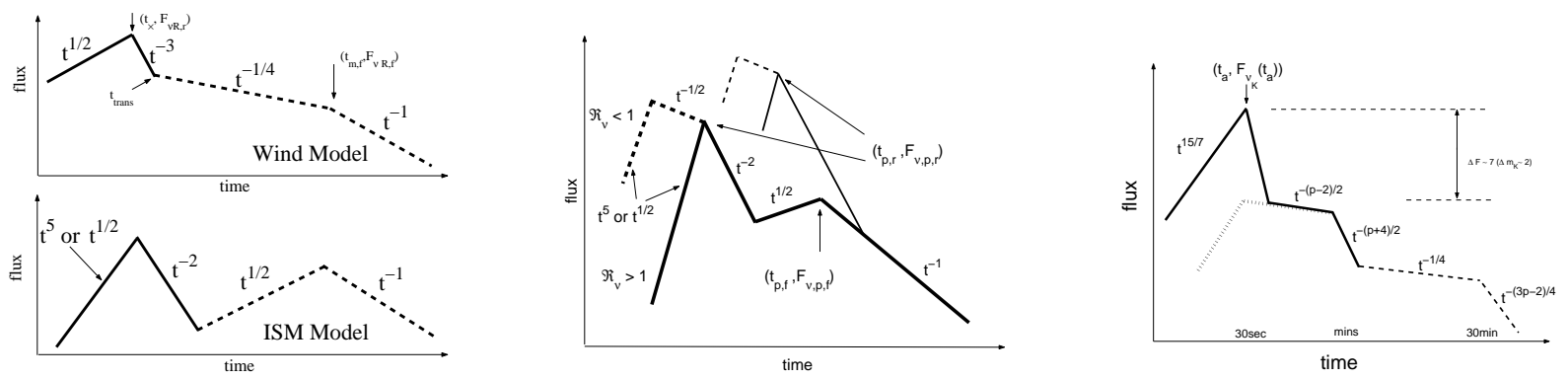

FIGURE 1. Left panel: Typical early optical afterglow lightcurves for the ISM (bottom) and the wind (top) cases [7]. Solid lines are for the reverse shock component, and the dashed lines are for the foreward shock emssion component; Middle Panel: Two types early optical afterglow lightcurves for the ISM case[11]. Type I (thick line) is the rebrightening type, with a distinct separation of the reverse and forward shock components. Type II (thin line) is the flattening type, with the forward shock peak burried beneath the reverse shock component. A Type II lightcurve is usually associated with a strongly magnetized fireball; Right panel: Another typical optical/IR lightcurve for the wind case[12]. This is applicable when the inverse Compton scattering is not important in the reverse shock region.

For the ISM case, the early afterglow lightcurves could be further categorized into two types[11] (see the middle panel of Fig. 11). For typical parameters and assuming that the shock parameters (equipartition parameters $\varepsilon_{e}$ and $\varepsilon_{B}$, as well as the power-law index of the particle distribution $p$ ) are the same in both shocks, the lightcurve (Type I) is characterized by a "re-brightening" feature, i.e., there are two distinct lightcurve peaks for both shocks. Conversely, under certain conditions, the forward shock peak is burried beneath the reverse shock emission component, and the lightcurve (Type II) is characterized by a "flattening" feature. A Type-II lightcurve usually requires a stronger magnetic field in the reverse shock region than in the forward shock region, i.e., refers to a strongly magnetized central engine.

For the wind case, the early afterglow lightcurves could be also further categorized into two types based on whether synchrotron self-inverse Compton (IC) emission is important. A wind-type medium implies a low cooling frequency and a high selfabsorption frequency. The synchrotron self-absorption effect prevents the electrons from cooling, so that electrons are potentially piled up near the self-absorption energy[12]. If the IC effect is important, this additional cooling mechanism tends to destroy the pile-up bump, so that the treatment that neglects it gives the (approximately) correct description of the lightcurves[7] (top lightcurve, left panel, Fig. 11). If the IC cooling is less important compared with the synchrotron cooling, as is expected for a strongly magnetized central engine[11], the electron pile-up effect is prominent, which implies a bump in the synchrotron spectrum and hence, another bump in the early afterglow lightcurve[12] (see right panel, Fig. 11). Detections of such a bump would provide valuable information to estimate the wind mass lose and other fireball parameters[12]. 


\section{A Strongly Magnetized GRB Central Engine}

Early afterglow lightcurves could be used to constrain important fireball parameters, such as the initial Lorentz factor, wind mass loss, etc[11, 7, 12]. Another important piece of information is about the magnetic content of the fireball. A strongly magnetized central engine is widely speculated to power GRBs on many grounds (e.g. [13] for a review). If this is the case, the magnetic field in the reverse shock region is expected to be stronger than that in the forward shock region, since the fireball itself would carry some fields from the central engine. Defining $R_{B}=B_{r} / B_{f}$ as a free parameter (where $B_{r}$ and $B_{f}$ are the magnetic field strengths in the reverse shock and forward shock, respectively), one can use the early afterglow lightcurves to constrain $R_{B}[11]$. Using a straightforward analysis by combining both the reverse and the forward shock emission data, we have performed detailed case studies for the GRBs that have early afterglow detections. The results suggest that $R_{B}$ is larger than unity for both GRB 990123 and GRB 021211[11]. The latter result is confirmed by a more detailed, independent study[14]. These results suggest that at least for some bursts, the central engine is likely entrained with strong magnetic fields. The discovery of strong linear polarization[15] of gamma-ray emission in GRB 021206 is also consistent with such a picture.

An important question is how strong the magnetic energy density is as compared with the kinetic energy density. Conventionally one can define $\sigma=L_{P} / L_{K}$ to categorize the fireball (where $L_{P}$ and $L_{K}$ are the Poynting flux luminosity and kinetic energy luminosity, respectively). The canonical fireball is in the $\sigma \ll 1$ regime. For GRB 990123, broadband modeling suggests that $\varepsilon_{B, f} \sim 7.4 \times 10^{-4}[16]$. Our analysis indicates that $R_{B}=$ $\left(\varepsilon_{B, r} / \varepsilon_{B, f}\right) \sim 15$ for this burst, so that $\varepsilon_{B, r} \sim 0.17$. This is still in the $\sigma \ll 1$ regime, which ensures self-consistency of our hydrodynamical treatment. In the meantime, it suggests that the magnetitized fireball is not Poynting-flux dominated at the deceleration radius.

\section{A QUASI-UNIVERSAL STRUCTURED JET MODEL}

\section{Uniform vs. Universal Jets}

One intriguing finding in the GRB afterglow observations is that the geometrycorrected total energy for different bursts is standard[17, 18]. There are two equivalent interpretations. One is that different GRBs collimate a same amount of energy in different solid angles, but with a uniform energy distribution within the jets. The other is that all GRBs have a same jet configuration, but the energy per solid angle decreases with angle from the jet axis in the form of $\varepsilon(\theta) \propto \theta^{-2}$, so that the inferred jet angles from the afterglow data correspond to the observers' viewing angles[19, 20]. The former is called "uniform jets", and the latter is called "universal jets".

These two models are two extremal presentations of what might happen in reality. In realistic simulations such as those in the collapsar model, the emerging GRB jets natually have a non-uniform angular structure[21]. On the other hand, it is unrealistic to expect that all GRB jets are exactly universal. Such an exactly universal picture is 
already disfavored by the $\log \left(E_{i s o}\right)-\log \left(\theta_{j}\right)$ plot of the observed data, which indicate a large scatter around the $E_{i s o} \propto \theta_{j}^{-2}$ line (see solid squares in Fig. 2).

\section{Quasi-Universal Jets: Power Law vs. Gaussian}

A reasonable picture is that GRB jets preserve certain angular structure individually, and may have a "quasi-universal" pattern of the jet structure[22, 23]. The so-called quasi-universal jet model suggests that all GRBs have a more-or-less similar angular jet structure, with the model parameters (e.g. the power-law index for the power-law jets, the typical angle for the Gaussian jets, and the normalization parameters for both types of jets) being distributed around some standard values with a small scatter.
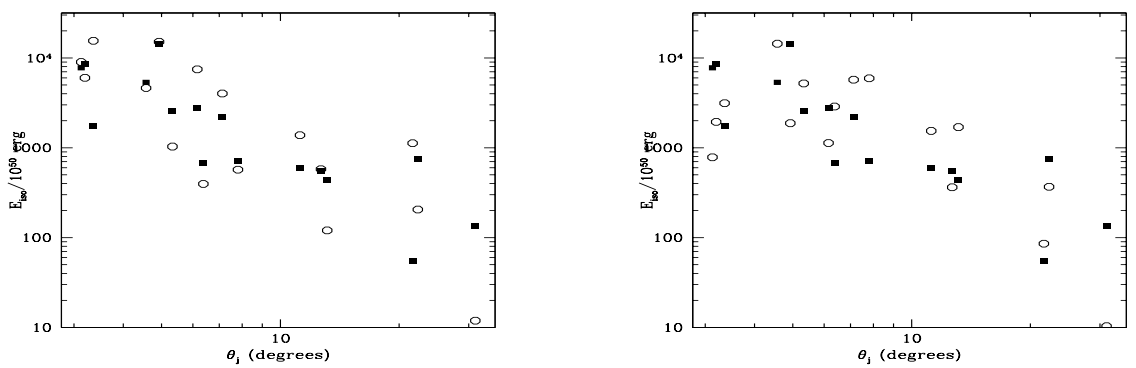

FIGURE 2. Simulated $E_{i s o}-\theta_{j}$ data from the quasi-universal structured jet models [22] (open circles) as compared with the data[18] (solid squares). Left panel: Quasi-universal power-law model; Right panel: Quasi-universal Gaussian model.

When parameters are allowed to have some scatter, the $k=-2$ power law structure is no longer a pre-requisite for individual bursts. Other types of jet structure (such as Gaussian)[20] are also allowed, especially when the total energy within the jet is preserved to be a quasi-constant. Figure 2 shows that both a quasi-universal power-law model and a quasi-universal Gaussian model can reproduce the $E_{i s o}-\theta_{j}$ data[22].

\section{Quasi-Universal Gaussian Jets: A Unified Model for GRBs and XRFs}

X-ray flashes (XRFs) are the natural extension of GRBs towards the softer and fainter regime. Recent HETE-2 data reveal that an intriguing empirical relation $E_{p} \propto\left(E_{i s o}\right)^{1 / 2}$ (where $E_{p}$ is the peak energy of the GRB-XRF spectrum) [24] is extended from GRBs to XRFs[25], and that the number ratio among GRBs, X-ray rich GRBs (XRGRBs) and XRFs is roughly $1: 1: 1$. These facts pose severe constraints on both the universal[25] and the uniform[23] jet models. The current GRB-XRF prompt emission and afterglow data are, however, consistent with a quasi-universal Gaussian jet model[23].

Figure 3 presents some predictions of the quasi-universal Gaussian jet model. The GRB luminosity function (left panel) is predicted to be a broken power-law with indices changing from -1 to $\sim-2[22]$. This is consistent with some luminosity function studies. The GRB:XRGRB:XRF number ratio is roughly 1:1:1 (middle panel), and the afterglow 

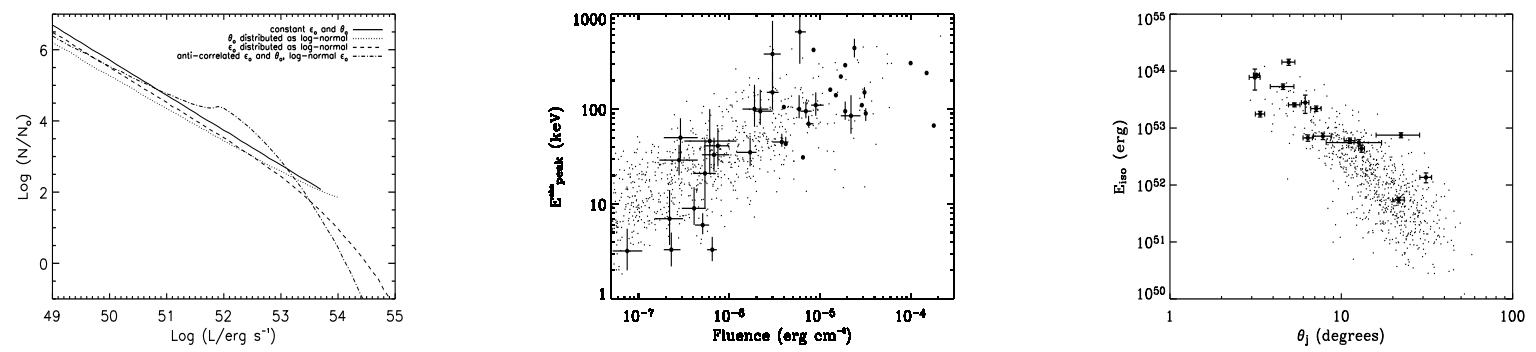

FIGURE 3. A quasi-universal Gaussian jet model confronted with the current data. Left: the predicted GRB luminosity functions 22]; Middle: the $E_{p}$-fluence diagram[23]; Right: the $E_{i s o}-\theta_{j}$ diagram[23].

$E_{i s o}-\theta_{j}$ correlation is consistent with the "standard energy reservoir" relation[23]. More rigorous tests of this model with a wider spectrum of data are being performed[26].

\section{ACKNOWLEDGMENTS}

This research is supported partly through NASA NAG5-13286, NSF PHY 01-14375, and the Monell Foundation.

\section{REFERENCES}

1. Mészáros, P. \& Rees, M. J. ApJ, 476, 232 (1997)

2. Sari, R. \& Piran, T. ApJ, 517, L109 (1999)

3. Kobayashi, S. ApJ, 545, 807 (2000)

4. Akerlof, C. W. Nature, 398, 400 (1999)

5. Fox, D. et al. Nature, 422, 284 (2003)

6. Li, W. et al. ApJ, 586, L9 (2003)

7. Kobayashi, S. \& Zhang, B. ApJ, 597, 455 (2003)

8. Sari, R., Piran, T. \& Narayan, R. ApJ, 497, L17 (1998)

9. Kobayashi, S. \& Zhang, B. ApJ, 582, L75 (2003)

10. Chevalier, R. A. \& Li, Z.-Y. ApJ, 520, L29 (1999)

11. Zhang, B., Kobayashi, S. \& Mészáros, P. ApJ, 595, 950 (2003)

12. Kobayashi, S. Mészáros, P. \& Zhang, B. ApJL, in press (astro-ph/0308409) (2003)

13. Zhang, B. \& Mészáros, P. Int. J. Mod. Phys. A, in press (astro-ph/0311321) (2003)

14. Kumar, P. \& Panaitescu, A. MNRAS, in press (astro-ph/0305446) (2003)

15. Coburn, W. \& Boggs, S. E. Nature, 423, 415 (2003)

16. Panaitescu, A. \& Kumar, P. ApJ, 571, 779 (2003)

17. Frail, D. et al. ApJ, 562, L155 (2001)

18. Bloom, J., Frail, D. A. \& Kulkarni, S. ApJ, 594, 674 (2003)

19. Rossi, E., Lazzati, D. \& Rees, M. J. MNRAS, 332, 945 (2002)

20. Zhang, B. \& Mészáros, P. ApJ, 571, 876 (2002)

21. Zhang, W., Woosley, S. E. \& MacFadyen, A. I. ApJ, 586, 356 (2003)

22. Lloyd-Ronning, N. M., Dai, X. \& Zhang, B. ApJ, in press (astro-ph/0310431) (2003)

23. Zhang, B., Dai, X., Lloyd-Ronning, N. M. \& Mészáros, P. ApJL, in press (astro-ph/0311190) (2003)

24. Amati, L. et al. A\&A, 390, 81 (2002)

25. Lamb, D. Q., Donaghy, T. Q. \& Graziani, C. astro-ph/0309456 (2003)

26. X. Dai and B. Zhang, in preparation (2003) 